\title{
Miranda
}

Revue pluridisciplinaire du monde anglophone /

Multidisciplinary peer-reviewed journal on the English-

speaking world

16 | 2018

L'expérimental dans la littérature et les arts

contemporains

\section{Sara Greaves, Côté guerre Côté jardin. Excursions dans la poésie de James Fenton}

Isabelle Keller-Privat

\section{OpenEdition}

Journals

Édition électronique

URL : http://journals.openedition.org/miranda/11492

DOI : 10.4000/miranda. 11492

ISSN : 2108-6559

Éditeur

Université Toulouse - Jean Jaurès

Référence électronique

Isabelle Keller-Privat, « Sara Greaves, Côté guerre Côté jardin. Excursions dans la poésie de James Fenton », Miranda [En ligne], 16 | 2018, mis en ligne le 05 juin 2018, consulté le 16 février 2021. URL http://journals.openedition.org/miranda/11492 ; DOI : https://doi.org/10.4000/miranda.11492

Ce document a été généré automatiquement le 16 février 2021.

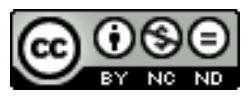

Miranda is licensed under a Creative Commons Attribution-NonCommercial-NoDerivatives 4.0 International License. 


\title{
Sara Greaves, Côté guerre Côté jardin. Excursions dans la poésie de James Fenton
}

\author{
Isabelle Keller-Privat
}

\section{RÉFÉRENCE}

Sara Greaves, Côté guerre Côté jardin. Excursions dans la poésie de James Fenton (Aix-enProvence : Presses universitaires de Provence, 2016), 240 p., ISBN 979-10-320-0074-8

1 Dans son ouvrage Côté guerre Côté jardin. Excursions dans la poésie de James Fenton, publié aux Presses Universitaires de Provence, Sara Greaves offre au lecteur un voyage tant littéraire qu'historique dans une œuvre poétique qui se fait témoin de son temps et dont la lecture s'avère essentielle pour la compréhension de notre société contemporaine. James Fenton, né en 1949, et connu à la fois comme poète, critique et journaliste, fut en effet aussi un grand voyageur qui témoigna du retrait des troupes américaines du Vietnam, ou encore de la montée au pouvoir de Pol Pot au Cambodge. Sa poésie retrace ainsi l'histoire d'une conscience politique qui se confronte douloureusement au monde.

2 L'ouvrage de Sara Greaves se divise en trois grandes parties : l'analyse de l'œuvre de Fenton occupe tout d'abord trois chapitres. Viennent ensuite des extraits de l'œuvre de Fenton dont l'auteur propose à chaque fois une traduction en regard du texte anglais. Enfin, la dernière partie consacrée aux « Notes traductologiques » éclaire le lecteur sur la méthode et les stratégies de la traductrice.

3 L'ensemble de l'ouvrage relève d'une véritable gageure : rendre compte de la richesse et de la diversité d'une œuvre à la fois poétique et critique à travers un travail qui conjoint la rigueur de l'analyse et la perspicacité créatrice de la traduction.

4 C'est avec une grande attention aux mouvements littéraires et aux grands courants idéologiques que Sara Greaves commence, dans son premier chapitre «Front et 
Arrière " par redéfinir la "war poetry » (7) tout en soulignant en quoi le concept même de poésie de guerre est problématique et participe d'une "transmission de la culpabilité » (9). En interrogeant le rapport de James Fenton à l'Histoire (celle du Vietnam, du Cambodge, des Philippines mais aussi des États-Unis et de l'Angleterre), Sara Greaves révèle un personnage à la fois humaniste et polyvalent dont le regard sait percer l'hypocrisie de son époque pour dénoncer la fausse fin de l'impérialisme et l'insistance larvée de la violence sociale. Sara Greaves montre avec doigté comment James Fenton tisse une pensée du politique et du poétique en assignant à la poésie la fonction d'une «méditation libérée [...] vers les découvertes les plus improbables.» (16) Un nouveau rapport au prosaïque émerge des procédés textuels et stylistiques qui viennent interroger notre rapport intime à la violence omniprésente et insidieuse de l'Histoire.

Explorant les rapports de culpabilité de Fenton à l'Histoire du Cambodge, Sara Greaves montre comment la poésie engagée déploie une esthétique de l'empathie et du non-dit qui participe d'une poét(h)ique du sensible. Or, il semble que Sara Greaves pourrait peut-être interroger davantage encore cette "expérience paradoxale [du] témoin second» (29), et la question de la transmission de l'expérience collective et du dessaisissement du «je». S'amorce en effet ici une réflexion porteuse qui évoque le travail de Jean-Christophe Bailly sur « la scène pronominale » et sur le « Nous » dans son récent ouvrage L'élargissement du poème (Paris : Christian Bourgeois Editeur, 2015). Dans cette optique, le témoignage décentré que porte l'écriture de Fenton se donnerait à lire non comme « une vague empathie universelle qui, à nouveau, formerait un cercle, une étendue de compatibilité mais comme [...] une surface sans bords et en tout cas incapable de faire masse ou famille ou giron: surface d'apparition des intensités, surface de passage des connexions [...] » (Bailly, 166).

6 À l'appui de la théorie, Sara Greaves convoque des textes qu'elle sait toujours analyser avec une subtilité et une élégance qui révèlent l'œil aiguisé et la sensibilité de la traductrice. Elle trouve chez Fenton un poète dont l'art minimaliste fait éclore de nouveaux rapports, tout comme ceux qu'elle sait habilement nouer entre la langue française et anglaise. Ainsi, les micro-lectures de "A German Requiem », «Jerusalem », "Out of the East» ou de "The Manila Manifesto » sont particulièrement éclairantes tant sur la facture propre à chaque texte que sur l'esthétique du poète et la savante élaboration de ce que Sara Greaves appelle «le 'dispositif' du poème fentonien » (50). L'analyse stylistique et prosodique s'articule parfaitement avec le questionnement théorique qui montre comment Fenton redessine notre conception du témoignage, de l'expérience du quotidien et du politique.

7 Le deuxième chapitre, intitulé «Retour au Même» définit précisément le dispositif propre à la poétique de Fenton au sein de laquelle l'hétérogénéité devient le principe même du renouvellement de l'écriture. Suite logique de la démonstration du chapitre précédent qui s'est attaché à démontrer les liens entre "Front et Arrière », ce deuxième chapitre montre comment la poésie de Fenton se lit comme un mouvement d'inclusion de la langue qui s'ouvre au commun et prend ses distances avec le discours idéologique. Les procédés de «narration secrète» (52), de balancement et de décentrement participent d'une rhétorique qui interroge l'essence du langage poétique. C'est ce qui conduit Sara Greaves à retracer à travers ses analyses des poèmes de Fenton la longue chaîne de poètes qui passe par Milton, Apollinaire, Basil Bunting, W. H. Auden ou Ted 
Hughes et qui permet d'éclairer le texte dans ses anfractuosités les plus infimes, comme par exemple lors de la splendide analyse du terme « dismay ».

8 Le troisième chapitre intitulé "La 'Voix-peau' » choisit un angle psychanalytique qui permet à l'auteur de travailler la " dimension collective de la lecture » (79) qu'appelle le texte de Fenton. Cette approche offre une lecture éclairante de la place de l'enfant dans la poésie de Fenton, tout en permettant également d'inscrire le poète dans la longue tradition romantique de Blake à Wordsworth, et d'ouvrir de nouvelles pistes de réflexion sur le concept de voix, sur son inscription, sur ses modulations et sur son déploiement particulier dans l'écriture de Fenton.

Enfin, la rapide conclusion qui réaffirme les spécificités de cette œuvre, à la fois critique et poétique, et revendique son appartenance au canon de la littérature britannique surprend le lecteur qui, porté par cette « voix-levée », ce « désir de se faire entendre» (90) pressent ici les résonnances contemporaines de cette écriture qui ravive une sensibilité perdue et tente de refonder une communauté poétique et humaine vibrante.

10 Les textes choisis et proposés en version bilingue par l'auteur ainsi que les commentaires traductologiques qui suivent proposent au lecteur un magnifique parcours dans une œuvre encore trop méconnue. Sara Greaves a réussi son pari en composant un ouvrage qui donne à la fois envie de comprendre et d'éprouver la poésie de James Fenton. L'aisance avec laquelle elle manipule les concepts théoriques épargne toujours au lecteur la raideur du jargon et donne lieu à de délicieuses trouvailles, comme celle du terme "peau-éthique » (93). Les outils critiques fonctionnent comme autant de ponts vers une réception sensible de l'œuvre ; sa maîtrise de la traduction, conçue à la fois comme une réflexion théorique et comme une œuvre de transmission, est à la hauteur du défi qu'elle s'est lancée et qu'elle sait partager avec son lecteur. Le choix du dernier poème est particulièrement éloquent qui convoque à la toute fin de l'ouvrage le désir de laisser parler encore un peu les lèvres muettes des tulipes jaunes et qui rassemble dans un même échange silencieux le poète et son lecteur :

I can see the lips, parted first in surprise, parted in desire,

Smile now as a silence falls on the yellow-dappled ride

For each thinks the other can hear each receding thought

On each receding tide. (204) 


\section{INDEX}

Keywords : contemporary poetry, war poetry, criticism, post-imperial, postcolonial, collage, violence, minimalism, travel narrative, guilt, empathy, testimony, indetermination, collective experience, poetics and politics, identity, margins, hybridity, tradition, aesthetics and ethics, city, childhood, Holocaust, mourning, psychoanalysis, transitional space, trauma, body, sensibility, humanism, stylistics, translation, prosody, orality

Mots-clés : poésie contemporaine, poésie de guerre, critique, post-empire, postcolonial, collage, violence, minimalisme, récit de voyage, culpabilité, empathie, témoignage, indétermination, expérience collective, poétique et politique, identité, marges, hybridité, tradition, esthétique et éthique, villes, enfance, Holocauste, deuil, psychanalyse, espace transitionnel, trauma, corps, sensibilité, humanisme, stylistique, traduction, prosodie, oralité

\section{AUTEURS}

\section{ISABELLE KELLER-PRIVAT}

Maître de conférences HDR

Université Toulouse II - Jean Jaurès

isa.kellerprivat@free.fr 